\title{
\begin{tabular}{l|l} 
pcori). & PATIENT-CENTERED OUTCOMES RESEARCH INSTITUTE \\
RESEARCH SUMMARY
\end{tabular}
}

\section{Improving Methods for Discrete Choice Experiments to Measure Patient Preferences}

Principal investigator

Alan R. Ellis, PhD, MSW
Organization

North Carolina State University

\section{What was the project about?}

Researchers can use experiments to learn about what patients prefer. Discrete choice experiments, or DCEs, describe treatments with different features, such as out-of-pocket costs or wait times. Patients fill out surveys about which treatments they prefer. From their choices, researchers learn what is most important to patients and how they think about the different features.

DCEs can be hard to design and analyze. When surveys are complex, patients may ignore information or take shortcuts, which leads to inaccurate results.

To make DCE results more accurate, researchers can

- Change the design of the DCE

- Apply statistical methods

But current knowledge of how to do this is limited.

In this project, the research team looked at improving methods to design and analyze DCEs.

\section{What did the research team do?}

First, the research team looked at how changes to the design of the DCE affected results. Using a computer program and data from two DCEs, the team created test data for 100,000 patients. The team used the test data to see how changes in DCE design, such as the number of patients taking part, affected results. DCES are complex, so researchers often test the design in a small pilot study, which informs the design of the main study. The team also looked at how the changes in pilot study designs affected the accuracy of results from the main studies.

Next, the research team looked at one type of statistical method used in DCEs called random parameter logit estimation with Halton draws. This method lets researchers measure what patients prefer while accounting for different preferences across patients. The team tested the method under different conditions, such as how much preferences vary from patient to patient. Then they looked at how many Halton draws were needed to get accurate results in a DCE study.

The research team worked with other DCE researchers to design this study.

\section{What were the results?}

When the DCE design included more patients, the results were more accurate for assessing patient preferences. If the pilot study had design errors, results from the main study were less accurate.

In random parameter logit estimation with Halton draws, the research team figured out the number of Halton draws needed to improve the accuracy of DCE results. 


\section{What were the limits of the project?}

The research team used two DCEs and varied a few study design aspects. Results may differ for other data sets and design changes.

Future research could test the random parameter logit estimation with Halton draws with other data sets and designs.

\section{How can people use the results?}

Researchers can use the results to improve how they design and analyze DCEs.

To learn more about this project, visit www.pcori.org/Ellis346. 